\title{
Perfil epidemiológico da mortalidade materna no Brasil no período de 2015 a 2019
}

\author{
Epidemiological profile of maternal \\ mortality in Brazil from 2015 to 2019
}

Bianca Leão Barreto

Faculdade de Ciências Humanas de Olinda (Olinda). Pernambuco, Brasil. bianquinhaleao@gmail.com

RESUMO | OBJETIVO: Determinar o perfil epidemiológico dos óbitos maternos no Brasil no período de 2015 a 2019. METODOLOGIA: Estudo epidemiológico descritivo retrospectivo com dados extraídos na base de dados do TABNET/DATASUS. As variáveis de interesse foram: ano de notificação, região de notificação, faixa etária, cor/raça, escolaridade, estado civil, local de ocorrência e tipo de causa obstétrica. Os dados foram analisados por estatística descritiva, por meio de frequência absoluta e relativa. RESULTADOS: No período, o total de óbitos foi de 324.792. O ano que apresentou o maior registro de óbitos foi 2016 (67.147) e a região foi a Sudeste (136.012). Com relação à faixa etária, houve maior frequência na idade de 40 a 49 anos (48,5\%). Observou-se predomínio da cor/raça parda $(45,9 \%)$ e branca $(40,8 \%)$, escolaridade de 8 a $11(28,4 \%)$ anos de estudo e estado civil solteira $(53,4 \%)$. A maioria dos óbitos ocorreu no hospital $(70,2 \%)$ e a principal causa foram as obstétricas indiretas $(73,1 \%)$. CONCLUSÃO: A mortalidade materna continua sendo um problema de saúde no Brasil, necessitando-se que alcance os níveis aceitáveis dos óbitos maternos estabelecidos pelos Objetivos de Desenvolvimento Sustentável (ODS). Portanto, é indispensável condutas que visem à redução da mortalidade, promovendo o planejamento familiar e uma assistência pré-natal de excelência.

DESCRITORES: Mortalidade materna. Mortalidade. Óbito. Saúde da mulher. Epidemiologia.

\begin{abstract}
OBJECTIVE: To determine the epidemiological profile of maternal deaths in Brazil from 2015 to 2019. METHODOLOGY: A retrospective descriptive epidemiological study with data extracted from the TABNET / DATASUS database. The variables of interest were: year of notification, region of notification, age group, color/race, education, marital status, place of occurrence, and type of obstetric cause. The data were analyzed using descriptive statistics, using absolute and relative frequency. RESULTS: The Southeast and Northeast regions had more deaths in 2016, followed by 2015 and 2017. There was a higher frequency in the age group of 30 to 39 and 40 to 49 years regarding the age group. There was a predominance of brown/white color/race, schooling from 4 to 7 and 8 to 11 years of study, and with marital status, single women. Most deaths occurred in the hospital, and the main cause was indirect obstetrics. CONCLUSION: The data indicate that maternal mortality remains a health problem in Brazil, requiring that it reach the acceptable levels of maternal deaths established by the SDGs. Therefore, it is essential to reduce mortality, promoting family planning and excellent prenatal care.
\end{abstract}

DESCRIPTORS: Maternal mortality. Mortality. Death. Women's health. Epidemiology. 


\section{Introdução}

A Organização Mundial de Saúde (OMS), na 10ª revisão da Classificação Internacional de Doenças (CID10), definiu que mortalidade materna é todo óbito que ocorre no período gestacional ou até 42 dias após o parto, não havendo influência sobre a localização da gestação ou duração da gravidez, relacionada à circunstância decorrente da gravidez ou agravada por ela. Não é considerada morte materna a que é provocada por fatores acidentais ou incidentais ${ }^{1}$.

A Razão de Mortalidade Materna (RMM) é um indicador que analisa as mudanças demográficas, geográficas e temporais dos óbitos maternos, identificando os casos de desigualdade e as demandas de ações específicas². A mortalidade materna é indicativo importante na qualidade de vida da população, pois grande parte das mortes são precoces e evitáveis, atingindo a população com baixo nível socioeconômico, refletindo sobre a desorganização, a desarticulação, a desigualdade de gênero e baixa qualidade da assistência nos serviços de saúde prestada a esse grupo 3 .

Em 2015, as Nações Unidas estabeleceram os novos Objetivos de Desenvolvimento Sustentável (ODS) de 2016-2030, uma das metas é reduzir a taxa de mortalidade materna global para menos de 70 por cada 100 mil nascidos vivos. O Brasil propôs como meta nacional a redução para no máximo 20 mortes por 100 mil nascidos vivos até o ano de 20304.

No presente momento, aproximadamente $830 \mathrm{mu}$ Iheres vão a óbitos todos os dias no mundo, por complicações evitáveis referente à gravidez ou ao parto. Por volta de 1990 a 2015, a taxa de mortalidade materna global reduziu apenas 2,3\% ao ano, em alguns países a redução anual foi maior que 5,5\%². No Brasil, em 2018, houve uma redução de $8,4 \%$ da RMM, no ano anterior era de 64,5 e foi para 59,1 . Neste período, as regiões que apresentaram maiores reduções da RMM foram Sudeste com 14,6\%, Norte com 9,1\%, e Nordeste com $8,3 \%$.
As mortes maternas são divididas em três grupos: obstétricas diretas, obstétricas indiretas e obstétricas indeterminada. A morte obstétrica direta é decorrente de complicações obstétricas, no período gestacional, parto ou puerpério, ocasionada devido a intervenções, omissões, tratamento incorreto ou a qualquer ocorrência resultante de algum dessas causas. A morte obstétrica indireta são aquelas decorrentes de doenças pré-existentes ou que desenvolveram durante a gestação, não causada por motivos obstétricos diretos, mas intensificados pelos efeitos fisiológicos da gravidez. As causas obstétricas indeterminada envolve as mortes por causa incidental ou acidental e ainda aquelas de causas diversas na gravide ${ }^{6}$.

A desigualdade nos serviços de saúde retrata o elevado número de óbitos maternos; $99 \%$ das mortes aconteceram nos países em desenvolvimento com ambientes frágeis e crises humanitárias. As principais causas em óbitos maternos nos países em desenvolvimento são em adolescentes menores de 15 anos, devido às complicações na gestação e no parto.

O Ministério da Saúde tem adotado estratégias para fortalecer e qualificar as ações no atendimento às gestantes, fazendo melhoria do pré-natal ao puerpério. As estratégias que ganharam visibilidade foram: a Rede Cegonha, a implantação e implementação do Plano de Redução da Mortalidade Materna e na Infância por Causas Evitáveis (PREMMICE) e a Estratégia Zero Morte Materna por Hemorragia, criada pela Organização Pan-Americana da Saúde (OPAS). Essas intervenções visam estabelecer medidas de orientação e qualificação dos profissionais que atuam na rede de atenção às gestantes e puérperas .

Por se tratar de um problema de saúde pública, que atinge principalmente os países em desenvolvimento, justifica-se a importância de estudar o perfil destas mulheres e as causas envolvidas, contribuindo para o planejamento das estratégias de saúde e redução dos óbitos maternos. Portanto, o presente artigo tem por objetivo determinar o perfil epidemiológico da mortalidade materna no Brasil, no período de 2015 a 2019. 


\section{Método}

Trata-se de um estudo epidemiológico descritivo. A elaboração da pesquisa foi realizada através de levantamentos dos dados secundários disponibilizados no item estatísticas vitais do TABNET/DATASUS. A coleta de dados ocorreu no ano de 2021. Foram utilizados os registros de mortalidade materna cadastrados no TABNET/DATASUS.

As variáveis utilizadas foram: ano de notificação, região de notificação, faixa etária, cor/raça, escolaridade, estado civil, local de ocorrência e tipo de causa obstétrica. Como critério de inclusão, foram considerados todos os óbitos em mulheres de idade fértil no território nacional, no período de 2015 a 2019.

A análise dos dados foi realizada por frequências absolutas e frequência relativa. Foram elaboradas tabelas no Microsoft Word 2016, possibilitando melhor visualização e análise dos dados.

O referido estudo não necessitou de liberação de Comitê de Ética e Pesquisa para a sua realização, pois utilizou dados secundários de domínio público.

\section{Resultados}

De acordo com análise da mortalidade nas mulheres em idade fértil, ocorreram 324.792 óbitos maternos no intervalo de cinco anos (2015-2019). Neste período, verificou-se que ocorreu uma maior notificação de óbitos na região do Sudeste com 136.012 (41,8\%) óbitos, seguido pelo Nordeste com 91.430 (28,2\%). Foi constatado que o ano que apresentou maior número de óbitos maternos foi em 2016, com 67.147 (20,7\%) das notificações, seguido de 2015 com 65.328 (20,1\%), havendo uma diminuição a partir de 2017 (Tabela 1).

Tabela 1. Óbitos maternos por regiões brasileiras no período de 2015 a 2019. Brasil. 2021

\begin{tabular}{|c|c|c|c|c|c|c|c|c|c|c|c|}
\hline \multirow{2}{*}{$\begin{array}{l}\text { Ano } \\
\text { Região }\end{array}$} & \multicolumn{2}{|c|}{2015} & \multicolumn{2}{|c|}{2016} & \multicolumn{2}{|c|}{2017} & \multicolumn{2}{|c|}{2018} & \multicolumn{2}{|c|}{2019} & \multirow{2}{*}{$\begin{array}{c}\text { Total } \\
\mathbf{n}\end{array}$} \\
\hline & $\mathbf{n}$ & $\%$ & $\mathbf{n}$ & $\%$ & n & $\%$ & n & $\%$ & n & $\%$ & \\
\hline Norte & 5.491 & 8,4 & 5.502 & 8,2 & 5.679 & 8,8 & 5.762 & 9,0 & 5.741 & 8,9 & 28.175 \\
\hline Nordeste & 18.188 & 27,8 & 18.917 & 28,2 & 18.394 & 28,6 & 17.968 & 28,2 & 17.963 & 28,0 & 91.430 \\
\hline Sudeste & 27.624 & 42,3 & 28.218 & 42,0 & 26.658 & 41,4 & 26.430 & 41,5 & 27.082 & 42,1 & 136.012 \\
\hline Sul & 8.921 & 13,7 & 9.216 & 13,7 & 8.762 & 13,6 & 8.508 & 13,4 & 8.520 & 13,3 & 43.927 \\
\hline $\begin{array}{l}\text { Centro- } \\
\text { Oeste }\end{array}$ & 5.104 & 7,8 & 5.294 & 7,9 & 4.873 & 7,6 & 5.025 & 7,9 & 4.952 & 7,7 & 25.248 \\
\hline Total & 65.328 & 100 & 67.147 & 100 & 64.366 & 100 & 63.693 & 100 & 64.258 & 100 & 324.792 \\
\hline
\end{tabular}

Na tabela 2, estão descritos os resultados dos registros de óbitos em mulheres de idade fértil de acordo com a faixa etária, cor/raça, escolaridade e estado civil no período analisado. Observou-se que as faixas etárias com maiores índices de mortalidade estão entre de 40 a 49 anos com 157.535 (48,5\%), e de 30 a 39 anos de idade, com 89.945 (27,6\%) óbitos. Segundo a variável de cor/raça, houve destaque dos casos com $149.046(45,9 \%)$ dos óbitos na cor/raça parda e em seguida a cor/raça branca com 132.382 (40,8\%). Com relação à escolaridade materna, houve maior frequência óbitos nas mulheres que tinham de 8 a 11 anos de estudo com 92.257 (28,4\%), seguida de 4 a 7 anos de estudo 77.829 (24,0\%). Destaca-se que 17,0\% das notificações não informavam o tempo estudado. No que tange ao estado civil, predominou registro de óbitos em mulheres solteiras $173.445(53,4 \%)$ óbitos. 
Tabela 2. Óbitos maternos de acordo com a faixa etária, cor/raça, escolaridade e estado civil no período de 2015 a 2019 , Brasil, 2021

\begin{tabular}{|c|c|c|}
\hline Variáveis & Total & $\%$ \\
\hline \multicolumn{3}{|l|}{ Faixa etária } \\
\hline 10 a 14 anos & 8.965 & $2,8 \%$ \\
\hline 15 a 19 anos & 18.298 & $5,6 \%$ \\
\hline 20 a 29 anos & 50.049 & $15,4 \%$ \\
\hline 30 a 39 anos & 89.945 & $27,6 \%$ \\
\hline 40 a 49 anos & 157.535 & $48,5 \%$ \\
\hline \multicolumn{3}{|l|}{ Cor/raça } \\
\hline Branca & 132.382 & $40,8 \%$ \\
\hline Parda & 149.046 & $45,9 \%$ \\
\hline Preta & 30.579 & $9,4 \%$ \\
\hline Amarela & 947 & $0,3 \%$ \\
\hline Indígena & 1.791 & $0,5 \%$ \\
\hline Ignorado & 10.047 & $3,1 \%$ \\
\hline \multicolumn{3}{|l|}{ Escolaridade } \\
\hline Nenhuma & 22.858 & $7,0 \%$ \\
\hline 1 a 3 anos & 44.437 & $13,7 \%$ \\
\hline 4 a 7 anos & 77.829 & $24,0 \%$ \\
\hline 8 a 11 anos & 92.257 & $28,4 \%$ \\
\hline 12 anos e mais & 31.206 & $9,6 \%$ \\
\hline Ignorado & 56.205 & $17,0 \%$ \\
\hline \multicolumn{3}{|l|}{ Estado civil } \\
\hline Solteiro & 173.445 & $53,4 \%$ \\
\hline Casado & 81.635 & $25,1 \%$ \\
\hline Viúvo & 7.138 & $2,2 \%$ \\
\hline Separado Judicialmente & 16.633 & $5,1 \%$ \\
\hline Outro & 22.858 & $7,0 \%$ \\
\hline Ignorado & 23.083 & $7,1 \%$ \\
\hline
\end{tabular}

Fonte: MS/SVS/CGIA - SIM.

Pode-se observar que no período analisado, o maior número de óbitos ocorreu no ambiente hospitalar 228.054 (70,2\%). Verificou-se que a causa dos óbitos que mais ocorreu foi a obstétrica indireta com 16.004 (73,1\%) (Tabela 3).

Tabela 3. Óbitos maternos com o local de ocorrência e tipo de causa obstétrica no período de 2015 a 2019 , Brasil, 2021

\begin{tabular}{lcc}
\hline Variáveis & Total & $\%$ \\
\hline Local de ocorrência & & \\
Hospital & 228.054 & $70,2 \%$ \\
Outro estabelecimento de saúde & 18.327 & $5,6 \%$ \\
Domicílio & 46.463 & $14,3 \%$ \\
Via pública & 19.763 & $6,1 \%$ \\
Outros & 11.891 & $3,7 \%$ \\
Ignorado & 294 & $0,1 \%$ \\
Tipo de causa & & $73,1 \%$ \\
Obstétrica indireta & 16.004 & $25,6 \%$ \\
Obstétrica direta & 5.607 & $1,2 \%$ \\
Obstétrica não especificada & 271 & \\
\hline Fonte: MS/SVS/CGIA SIM & & \\
\hline
\end{tabular}

Fonte: MS/SVS/CGIA - SIM. 


\section{Discussão}

A mortalidade materna é considerada evitável em 92\% dos casos, ocasionando uma grave violação dos direitos humanos das mulheres ${ }^{5}$. O índice elevado de mortalidade materna revela as características sociodemográficas de uma determinada localidade, refletindo nas diferenças sociais, nas condições de vida e serviços de saúde ofertados a população. Diante dos esforços realizados, o Brasil não alcançou a meta proposta nos ODS em 2015, havendo uma redução de $43 \%$ da $\mathrm{RMM}^{\stackrel{8}{ } \text {. }}$

O óbito materno, seja no decorrer da gestação, parto ou pós-parto, evidencia brechas nas diretrizes políticas da atenção à saúde da mulher, na equipe de saúde e na sociedade. As políticas de saúde proporcionam ações que em muitos casos não estão de acordo com suas necessidades, sendo necessário ter equidade da parte do serviço de saúde e da sociedade? Existem muitas dificuldades para oferta de serviço de saúde com qualidade quando a região possui poucos profissionais qualificados, impedindo a atenção na gestação e ao bebê. Outras condições que impedem as mulheres de receber ou procurar os cuidados são: a pobreza, a distância, a falta de informação, serviços inadequados e a práticas culturais ${ }^{4}$.

No período de 2015 a 2019, foram notificados 324.792 óbitos, os anos que tiveram os maiores números de mortes em ordem decrescente foram 2016, seguido por 2015, 2018, 2017 e 2019. Ao se tratar das regiões, Sudeste e Nordeste apresentaram domínio dos casos, e a região do Centro-Oeste apresentou a menor taxa de mortalidade. Os determinantes sociais tem total influência sobre a qualidade de vida de uma população. Desta maneira, as regiões que possuem níveis de pobreza vão ter índices elevados de destruição, anemia, violência, gravidez indesejada, precocidade da atividade sexual e escassez na qualidade de assistencial nos serviços de saúde ${ }^{10}$.

No Brasil, grande parte dos óbitos maternos ocorre na rede pública, demostrando escassez de estrutura física e de recursos materiais, falha nas políticas da atenção à saúde da mulher. A assistência prestada nos serviços de saúde utiliza medicações em excesso e o uso abusivo de intervenções desnecessárias, ocasionando uma situação obstétrica incabível, estando assim em contradição com os direitos das mulheres ${ }^{11}$.
De acordo com os dados coletados, os locais que mais acontecem os óbitos maternos são no ambiente hospitalar e no domiciliar. As mortes maternas ocasionadas pelos partos cesáreos evidenciam um alto risco comparado ao vaginal. É necessário a realização de um plano eficaz, abrangendo a qualidade no acesso ao serviço de saúde em tempo oportuno, a qualificação dos profissionais na identificação e tratamento dos casos de riscos ou urgência/emergência obstétrica, melhoria na assistência ao parto normal e redução das cesáreas desnecessárias ${ }^{11}$.

Neste estudo, a morte materna obstétrica indireta representou domínio dos óbitos em relação à morte materna obstétrica direta e à morte materna obstétrica não especificada; esses dados indicam que é necessário ter uma atenção na assistência prestada, tanto nas causas de morte relacionadas à gestação, quanto nas condições clínicas prévias à gestação. Nos países em desenvolvimento e os desenvolvidos, as justificativas dos óbitos obstétricos diretos têm mais peso do que os obstétricos indiretos, devido às patologias serem mais graves e apresentarem grande influência na gestação. As obstétricas diretas são mais evitáveis que as indiretas, sofrendo as consequências na qualidade da assistência no decorrer do ciclo gravídico-puerperal ${ }^{12}$.

Internacionalmente as mulheres em idade fértil são todas aquelas que estão na faixa etária de 15 a 49 anos, mas no Brasil essa faixa etária está entre 10 a 49 anos. Gestações precoces, muitas vezes no decorrer da adolescência, podem ser geradas devido à baixa escolaridade ou inexperiência para conquistar um emprego, podendo possibilitar uma união precoce, devido ao desejo ou pressão familiar ${ }^{5}$.

A faixa etária é um indicador importante para a mortalidade materna. No Brasil, evidenciou-se que houve uma frequência dos óbitos entre as mulheres de 30 a 39 anos e 40 a 49 anos, revelando que os maiores números de óbitos se dão entre as mulheres mais maduras. As gestações depois dos 35 anos apresentam maiores complicações obstétricas, apresentando riscos durante a gravidez, no parto com a presença da hemorragia, parto prematuro e má formação fetal. A análise espacial da mortalidade materna evidenciou que as mulheres acima de 30 anos de idade fazem parte do grupo vulnerável aumentando a probabilidade dos óbitos $\frac{13}{}$. 
No que se refere à escolaridade, evidenciou-se que houve uma maior ocorrência dos óbitos entre as mulheres que têm de 4 a 11 anos de estudo. A escolaridade também é um fator diretamente relacionando com a mortalidade materna. Quanto mais conhecimento se tem, a procura pelo serviço de saúde é maior. As mulheres que têm menos de 8 anos de estudo apresentam maior probabilidade de terem recém-nascidos com baixo peso ${ }^{14}$.

Ao se tratar da cor/raça, observa-se que houve predomínio da parda e da branca com mínima diferença. A cor/raça das mulheres mais afetadas são as pardas que possuem menor poder aquisitivo, têm poucas oportunidades nos serviços de saúde com acesso precário e limitado ao sistema. A questão da mortalidade não está restrita apenas aos serviços de saúde, mais contam com o fator biológico e o quadro clinico da mulher no momento do parto ${ }^{14}$.

O estado civil das mulheres de idade fértil que vão a óbito é bastante impressionante, onde todos os grupos são afetados, mas sofrem predomínio de dois grupos; o grupo das mulheres solteiras predomina. O estado civil precisa ser analisado, pois grande parte das relações matrimoniais leva ao rompimento do vínculo durante a gravidez. Portanto, acredita-se que as mulheres solteiras, viúvas ou separadas constituem grupos vulneráveis. $\mathrm{O}$ apoio do companheiro durante a gestação e puerpério demostra apoio emocional, segurança e suporte durante esse período ${ }^{15}$.

É essencial a mulher ser assistida por profissionais de saúde qualificados, que ofertem tratamento oportuno para a mãe e o bebê; desta maneira, as complicações criadas durante a gestação serão evitadas e tratadas, sendo importante a mãe ter cuidados prénatais durante a gestação, cuidados durante o parto e apoio no pós-parto. As complicações representam $75 \%$ de todas as mortes maternas, as principais são: hipertensão (pré-eclâmpsia e eclâmpsia); hemorragias graves (principalmente após o parto); infecções (normalmente depois do parto); complicações no parto; abortos inseguros ${ }^{4}$.

As limitações deste estudo estão relacionadas aos dados secundários. As notificações dos óbitos maternos no TABNET/DATASUS são realizadas através da subnotificação e sub-registros de mortalidade, portanto, devido à omissão da causa do óbito, perda dos dados, problemas de notificação, pendência de informações e/ou erros na coleta podem levar a inconsistências relacionadas à pesquisa. Outra limitação encontrada foi a identificação das causas dos óbitos, quando o sub-registro é preenchido de forma inadequada, mascaram a verdadeira causa do óbito, impossibilitando a identificação da morte como materna.

\section{Conclusão}

Este estudo revelou que, entre os anos de 2015 a 2019, o número de óbitos maternos no Brasil apresentou poucas alterações. Neste período, a região que obteve predomínio da mortalidade foi o Sudeste, e o ano que mais houve notificações foi 2016. Em relação às variáveis sociodemográficas, ocorreram mais óbitos entre mulheres na faixa etária de 40 a 49 anos de idade, com 8 a 11 anos de estudo, cor/raça parda e em mulheres solteiras. Com relação ao local dos óbitos, predominaram os casos de óbitos ocorridos no ambiente hospitalar e de causa relacionadas às obstétricas indiretas.

Conclui-se que a mortalidade materna continua sendo um problema de saúde no Brasil, necessitando-se que alcance os níveis aceitáveis dos óbitos maternos estabelecidos pelos ODS. Sendo indispensável a elaboração de condutas que visem a redução da mortalidade, promovendo o planejamento familiar e uma assistência pré-natal de excelência, dando ênfase na qualidade nos serviços de saúde visando minimizar os índices maternos através de uma equipe profissional qualificada para atendimento nas emergências obstétricas e observação no pós-parto.

\section{Conflitos de interesses}

\footnotetext{
Nenhum conflito financeiro, legal ou político envolvendo terceiros (governo, empresas e fundações privadas, etc.) foi declarado para nenhum aspecto do trabalho submetido (incluindo, mas não se limitando a subvenções e financiamentos, participação em conselho consultivo, desenho de estudo, preparação de manuscrito, análise estatística, etc.).
} 


\section{Referências}

1. Organização Mundial da Saúde. CID 10: Classificação Estatística Internacional de Doenças e Problemas Relacionados à Saúde. $8^{a}$ ed. São Paulo: Edusp; 2000.

2. Melanda SV, Larocca LM, Almeida WG, Oliveira CA, Nasr ACLF, Silva JCS. Sistematização da investigação do óbito materno: uma proposta de ferramenta para o monitoramento. Esp Saúde [Internet]. 2014;15(2):68-74. Disponível em: http:// espacoparasaude.fpp.edu.br/index.php/espacosaude/article/ view/53

3. Ministério da Saúde (Brasil), Secretaria de Atenção à Saúde, Departamento de ações programáticas estratégicas. Pacto nacional pela redução da mortalidade materna e neonatal [Internet]. Brasília: Ministério da saúde; 2004. Disponível em: https://portalarquivos.saude.gov.br/images/pdf/2016/ junho/20/2.a\%20Pacto\%20redu\%C3\%A7\%C3\%A30\%20 mortalidade.pdf

4. Organização Mundial da Saúde, Organização Pan-Americana da Saúde (Brasil). Folha informativa - Mortalidade materna [Internet]. Brasília: Organização Mundial da Saúde; 2018. [citado em 2021 fev 19]. Disponível em: https://www.paho.org/bra/ index.php?option=com_content \&view=article\&id=5741: follhainformativa-mortalidade-materna\&ltemid $=820$

5. Ministério da Saúde (Brasil), Secretaria de Vigilância em Saúde. Boletim epidemiológico. Monitoramento dos casos de arboviroses urbanas transmitidas pelo Aedes Aegypti (dengue, chikungunya e zika), semanas epidemiológicas 1 a 50, 2020 [Internet]. Vol 51. Brasília: Ministério da saúde; 2020. Disponível em: https://www. gov.br/saude/pt-br/media/pdf/2020/dezembro/28/boletim epidemiologico_svs_51.pdf

6. Governo do Estado do Ceará (Brasil), Secretaria de Saúde. Boletim epidemiológico: Mortalidade materna [Internet]. Ceará: Governo do Estado do Ceará; 2020. Disponível em: https://www. saude.ce.gov.br/wp-content/uploads/sites/9/2018/06/boletim epidemiologico_mortalidade_materna_n1_25112020.pdf

7. Ministério da Saúde (Brasil), Secretária da Atenção Primária à Saúde. Brasil reduziu 8,4\% a razão de mortalidade materna e investe em ações com foco na saúde da mulher [Internet]. Brasília: Ministério da Saúde; 2020. [citado em 2021 abr 4]. Disponível em: https://aps.saude.gov.br/noticia/8736
8. Marques CPC. Redes de atenção à saúde: A Rede Cegonha. São Luís: EDUFMA; 2016. 67 p.

9. Riquinho DL, Correia SG. Mortalidade materna: perfil sóciodemográfico e causal. Rev bras enferm. 2006;59(3):303-7. https:// doi.org/10.1590/S0034-71672006000300010

10. Governo do Estado do Ceará (Brasil), Secretaria da Saúde. Informe epidemiológico mortalidade materna [Internet]. Ceará: Secretária de Saúde do Ceará; 2014. Disponível em: https://www. saude.ce.gov.br/wp-content/uploads/sites/9/2018/06/informe mortalidade_materna_junho_2015.pdf

11. Silva SCM, Monteiro EA, Freitas WMF, Barros AG, Guimarães CMC, Melo SA. Diagnóstico da situação de morte materna. Rev Bras Promoç Saúde. 2019;32:9259. https://doi. org/10.5020/18061230.2019.9259

12. Ministério da Saúde (Brasil). Manual dos Comitês de Morte Materna. Brasília: Ministério da Saúde; 2007.

13. Wanderley RMM, Vasconcelos Neto PJA, Pinheiro HDM. Perfil da mortalidade materna. Rev enferm UFPE on line [Internet]. 2017;11(4):1616-24. Disponível em: https://periodicos.ufpe.br/ revistas/revistaenfermagem/article/download/15257/18050

14. Universidade Federal de Pernambuco. Especialistas do HC explicam a alta taxa de mortalidade infantil e materna superior na população preta e parda [Internet]. Recife: Universidade Federal de Pernambuco; 2020. [citado em 2021 fev 28]. Disponível em: https://www.ufpe.br/agencia/noticias/-/asset publisher/ dlhi8nsrz4hK/content/especialistas-do-hc-explicam-a-alta-taxa-demortalidade-infantil-e-materna-superior-na-populacao-preta-eparda/40615

15. Pereira LM. Mortalidade materna: como o descaso com a saúde da mulher impede a igualdade de gênero. Sau \& Transf Soc [Internet]. 2016;6(1):70-8. Disponível em: http:// incubadora.periodicos.ufsc.br/index.php/saudeetransformacao/ article/view/3144\#: :text=0\%20\%C3\%ADndice\%20de\%20 mortalidade $\% 20$ materna,das\%20mortes $\% 20$ poderia $\% 20$ ser $\% 20$ evitada 